American Journal of Applied Sciences 5 (4): 301-306, 2008

ISSN 1546-9239

(C) 2008 Science Publications

\title{
Mathematical Model to Simulate Tuberculosis Disease Population Dynamics
}

\author{
O.K. Koriko and T.T. Yusuf \\ Mathematical Sciences Department, Federal University of Technology, \\ Akure, Ondo state, Nigeria
}

\begin{abstract}
A mathematical model to depict Tuberculosis disease population dynamics was presented. The model population was compartmentalised as appropriate and the resulting model equations were solved numerically while different instances of the disease transmission were simulated. The graphical profiles of the various sub-populations with time were presented and discussed based on the results from our simulations. Also, the disease-free and endemic equilibrium of the system were established and analyzed for stability.
\end{abstract}

Keywords: Mathematical Model, Tuberculosis (TB), Directly Observed treatment Strategy (DOTS), Bacillus Calmette-Guerin (BCG), Stability analysis

\section{INTRODUCTION}

Tuberculosis (TB) is a contagious bacteria disease caused by inhaling the tubercle bacillus in the droplet nucleus form. Like the common cold, it spreads through the air. An infected person may have latent TB infection or active TB infection. Only actively infected people who are sick with TB bacilli in their lungs are infectious. When infectious people cough, sneeze, talk or spit, they propel TB germs, known as bacilli, into the air. A person needs only to inhale a small number of these to be infected ${ }^{[5]}$. A latent $\mathrm{TB}$ infected person does not show any symptoms of the disease and cannot infect others, though may live as long as possible without it degenerating into active TB.

Presently, the only effective vaccine for $\mathrm{TB}$ is Bacillus Calmette-Guerin (BCG) which is usually given to infants. This vaccine has a demonstrated efficacy ranging from no protection to $80 \%$ protection though a meta-analysis estimated that the overall efficacy of BCG is $50 \%^{[1]}$. However, due to case reports of disseminated BCG infection, where the vaccine is contraindicated in immunocompromised persons; safer and more efficacious vaccines are clearly needed ${ }^{[1]}$.

Christopher Murray ${ }^{[4]}$, Harvard School of Public Health, described a mathematical model developed to forecast the future impact of improvements in TB prevention and control. Specifically, this model projected the number of TB cases and deaths averted through the year 2050. Different scenarios were simulated to project the effect of adding TB vaccines to existing interventions. Six specific scenarios assessed the effect of vaccines (with efficacy levels of $20 \%$, $50 \%$, and $80 \%$ ) to protect from $M$. tuberculosis infection, as well as the effect of vaccines of the same levels of efficacy to protect latently infected persons from "breakdown" to active TB. Although a TB infection vaccine with $20 \%$ efficacy would prevent more than 30 million TB cases, the best protection is obtained from a TB breakdown vaccine with $80 \%$ efficacy, which would prevent almost 130 million TB cases. The breakdown vaccine could be used in the large number of persons with latent M. tuberculosis infection, now estimated at almost one third of the world's population. Such anticipated gains justify the effort to develop better TB vaccines.

In a related research work by Gammaitoni and Nucci ${ }^{[3]}$, where mathematical model was used to evaluate the efficacy of TB control measures, it was found that environmental control cannot eliminate the risk of TB transmission during high-risk procedures; respiratory protective devices, and particularly highefficiency particulate air masks may provide nearly complete protection if used with air filtration or ultraviolent irradiation, although the efficiency of these control measures decreases as the infectivity of the source case increases; while administrative control measures are the most effective because they substantially reduce the rate of infection .

In another research work conducted by Dye et al ${ }^{[2]}$, they developed a mathematical model that makes use of routinely-collected data to calculate the number of

\footnotetext{
Corresponding Author: $\quad$ Yusuf Tajudeen, Mathematical Sciences Department, Federal University of Technology, Akure, Nigeria
} 
deaths directly prevented by TB treatment. The method was applied to the world largest TB control programme covering over 500 million people in 12 provinces in China. Their results showed that counties which had enrolled in the programme since 1991 were, by 1997 , preventing at least $46 \%$ of the TB death that would have otherwise occurred. They concluded that the shortcourse chemotherapy has substantially reduced TB mortality in half of China.

According to the work of Castro ${ }^{[1]}$, the effect of the HIV epidemic on TB has been major in Africa, where HIV seroprevalence among TB cases is $50 \%$ to $70 \%$ and $\mathrm{TB}$ case notifications have at times tripled. However, countries with inadequate TB control are particularly exposed to the consequences of both epidemics. Moreover, he found out that TB remains an important public health problem in many areas of the world where direct observation treatment short-course (DOTS) has not been implemented because treatment outcomes were better in countries where DOTS has been used, though he averred that the strategy needs to be expanded rapidly and new tools to facilitate its implementation need to be developed.

The co-pandemic nature of TB-HIV calls for more quantitative research work on TB particularly because it has cure unlike HIV. It should then be no surprise that mathematical models are now been deployed in the study of the disease epidemiology and the evaluation of some of the world TB control measures and strategy. Thus, if we are able to reduce(to barest minimum) the spread of TB via all the control measures and strategies, we will have succeeded in reducing the alarming TB death tools and increase the life expectancy of HIV patients by reducing their chances of contacting TB, which may lead them to early grave.

In this paper, we will present a mathematical model for the Tuberculosis disease population dynamics using Susceptible-Infected-Recovered but Susceptible (SIRS) model which is different from the approaches used in some the cited paper, though results may be interrelated and can help to confirm or refute the earlier findings. Moreover, we shall evolve appropriate system of equations and solve the equations numerically. Also, we will simulate different instances of the infected subpopulation base on the effect of the different TB control measures and strategy. Finally, we shall establish the model system equilibriums and analyse their stability.

Model Formulation: Based on the standard SIRS model, the model population was compartmentalised into the susceptible (S) and the infected (I) which is further broken down into latently infected $\left(\mathrm{I}_{\mathrm{L}}\right)$ and actively infected $\left(I_{A}\right)$ while the recovered sub- population is ploughed back into the susceptible group due to the possibility of re-infection after successful treatment of the earlier infection. The model monitors the temporary dynamics in the population of susceptible people $\mathrm{S}(\mathrm{t})$, TB latently infected people $\mathrm{I}_{\mathrm{L}}(\mathrm{t})$ and $\mathrm{TB}$ actively infected people $\mathrm{I}_{\mathrm{A}}(\mathrm{t})$ as captured in the model system of ordinary differential equations that follows:

$$
\left.\begin{array}{l}
\frac{d S}{d t}=v f N-\alpha I_{A} S-\delta S+T_{A} I_{A}+T_{L} I_{L} \\
\frac{d I_{L}}{d t}=(1-p) \alpha I_{A} S-\beta_{A} I_{L}-T_{L} I_{L}-\delta I_{L} \\
\frac{d I_{A}}{d t}=p \alpha I_{A} S+\beta_{A} I_{L}-T_{A} I_{A}-\delta I_{A}-\varepsilon I_{A} \\
S(0)=N, I_{L}(0) \geq 0, I_{A}(0) \geq 0 .
\end{array}\right\}
$$

The model parameters and their respective

\begin{tabular}{|c|c|}
\hline Parameter & Description \\
\hline $\mathrm{N}$ & $\begin{array}{l}\text { Total number of new people into the } \\
\text { location of interest }\end{array}$ \\
\hline S & $\begin{array}{l}\text { Number of susceptible people in the } \\
\text { location }\end{array}$ \\
\hline $\mathrm{I}_{\mathrm{L}}$ & Number of TB latently infected people \\
\hline $\mathrm{I}_{\mathrm{A}}$ & Number of TB actively infected people \\
\hline$?$ & $\begin{array}{l}\text { Probability that a susceptible person is } \\
\text { not vaccinated }\end{array}$ \\
\hline $\mathrm{f}$ & Efficacy rate of vaccines \\
\hline $\mathrm{T}_{\mathrm{L}}$ & Success rate of latent TB therapy \\
\hline $\mathrm{T}_{\mathrm{A}}$ & Active TB treatment cure rate \\
\hline a & $\begin{array}{l}\text { TB instantaneous incidence rate per } \\
\text { susceptible }\end{array}$ \\
\hline d & Humans natural death rate \\
\hline $\mathrm{p}$ & $\begin{array}{l}\text { Proportion of infection instantaneously } \\
\text { degenerating into active TB }\end{array}$ \\
\hline $\mathrm{e}$ & TB-induced death rate \\
\hline$\beta_{\mathrm{A}}$ & $\begin{array}{l}\text { Breakdown rate from latent to active } \\
\text { TB }\end{array}$ \\
\hline
\end{tabular}
descriptions are given in the table below:

Table 1: Model parameters and their interpretations

As can be observed from (2.1), the susceptible population changes due to the coming of new susceptible people into the population $(v f N)$ where we assumed that people come into the location of interest at a constant rate $\mathrm{N}$ and this is further increased by the people who are cured from active TB and those successfully treated for latent TB while the susceptible population diminishes due to natural death at $\mathrm{d}$ death rate and infection with a incidence rate of infection. 
The instantaneous incidence rate of infection (a) per susceptible will depend on number of respiratory contact with infection per susceptible people and the probability that the contact lead to TB infection In same way, the latently infected population dynamics depends on the proportion of the TB infection that resulted in latent TB infection $\left((1-p) \alpha I_{A} S\right)$ and this is reduced by loss due to natural death, successful treatment of latent TB patients, and occasional breakdown of latent $\mathrm{TB}$ infection into active TB infection.

Finally, the change in the actively infected TB population is dependent on the proportion of TB infection of the susceptible sub-population degenerating instantaneously into active TB infection plus the number of latently infected people breaking down into active TB patients while this is diminished due to natural death, successful cure of active TB patients, and death caused as result chronic active TB infection.

It is important to note that in this model, we assumed that successfully treated latent TB patients and cured active TB patients becomes susceptible immediately after their treatments whenever they are expose to TB infection again irrespective of their TB infection history. Realistically, this group may sometimes enjoy some temporary immunity to TB infection and whenever they are re-infected, they may require more intensive treatment as a result of some multi-drug resistance that must have been built over time.

Equilibrium Analysis: Modelling infectious diseases demands that we investigate whether the disease spread could attain a pandemic level or it could be wiped out. The equilibrium analysis helps to achieve this. Thus, we shall consider the two equilibriums - the disease-free equilibrium and endemic equilibrium.

At equilibrium, the LHS of the three equations constituting the system of equation are zeros, i.e.

$\frac{d S}{d t}=0, \frac{d I_{L}}{d t}=0, \frac{d I_{A}}{d t}=0$.

Hence, the system of equation becomes:

$$
\left.\begin{array}{l}
v f N-\alpha I_{A} S-\delta S+T_{A} I_{A}+T_{L} I_{L}=0 \\
(1-p) \alpha I_{A} S-\beta_{A} I_{L}-T_{L} I_{L}-\delta I_{L}=0 \\
p \alpha I_{A} S+\beta_{A} I_{L}-T_{A} I_{A}-\delta I_{A}-\varepsilon I_{A}=0 \\
S(0)=N, I_{L}(0) \geq 0, I_{A}(0) \geq 0 .
\end{array}\right\}
$$

Obviously, at the disease-free situation $I_{L}=0$, and $I_{A}=0$.

Thus, from equation (3.1) we have $v f N-\delta S=0$.

$\Rightarrow S=\frac{v f N}{\delta}=N^{0}$

So, the disease-free equilibrium is $\left(N^{0}, 0,0\right)$ However, for the endemic equilibrium, we solve equation (3.1) for $\mathrm{S}, I_{L}$, and $I_{A}$ respectively. This gives

$$
\begin{aligned}
& S=\frac{v f N+T_{A} I_{A}+T_{L} I_{L}}{\delta+\alpha I_{A}}=S^{*}, \\
& I_{L}=\frac{(1-p) \alpha I_{A} S}{\beta_{A}+T_{L}+\delta}=I_{L}{ }^{*}, \\
& I_{A}=\frac{\beta_{A} I_{L}}{T_{A}+\varepsilon+\delta-p \alpha S}=I_{A}{ }^{*} .
\end{aligned}
$$

Thus, we can take our endemic equilibrium as $\left(S^{*}, I_{L}^{*}, I_{A}^{*}\right)$.

To test for the stability of each of the two equilibriums where we examine the behaviour of the model population near the equilibrium solutions (the disease free and the endemic), we need to linearize system of equations (2.1) by taking the Jacobian matrix of the system . For our system of equation (2.1), the Jacobian matrix is

$$
J=\left[\begin{array}{ccc}
-\left(\alpha I_{A}+\delta\right) & T_{L} & T_{A}-\alpha S \\
(1-p) \alpha I_{A} & -\left(\beta_{A}+T_{L}+\delta\right) & (1-p) \alpha S \\
p \alpha I_{A} & \beta_{A} & p \alpha S-\left(T_{A}+\varepsilon+\delta\right)
\end{array}\right]
$$

At the disease-free equilibrium $\left(N^{0}, 0,0\right)$, the Jacobian matrix becomes:

$$
J^{0}=\left[\begin{array}{ccc}
-\delta & T_{L} & T_{A}-\alpha N^{0} \\
0 & -\left(\beta_{A}+T_{L}+\delta\right) & (1-p) \alpha N^{0} \\
0 & \beta_{A} & p \alpha N^{0}-\left(T_{A}+\varepsilon+\delta\right)
\end{array}\right]
$$

We shall now obtained the eigenvalues of $J^{0}$ as below 


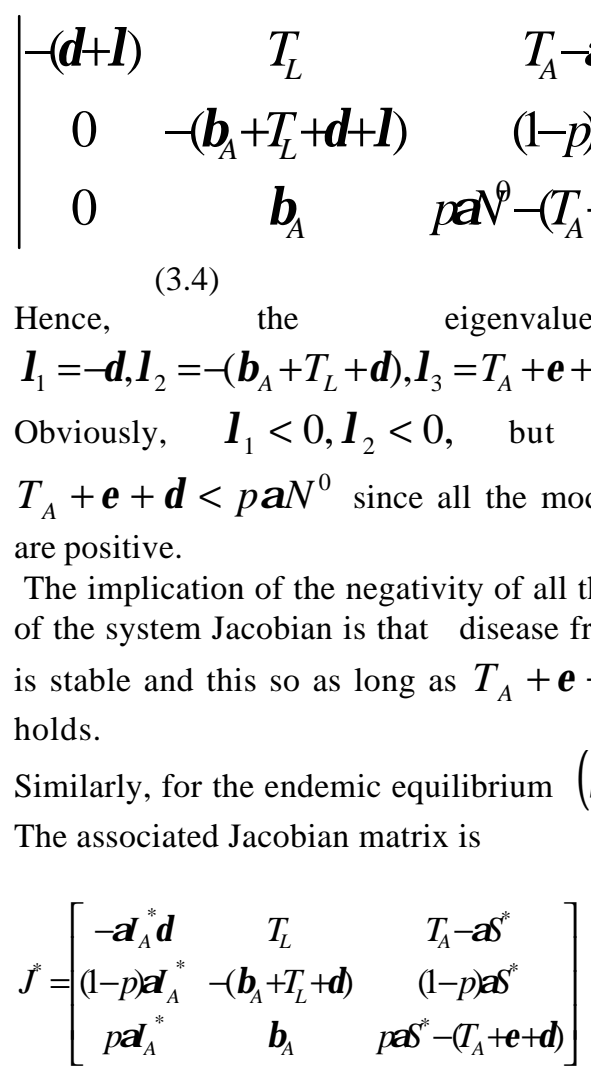

Obtaining the eigenvalues as usual from $\left|J^{*}-\lambda I\right|=0$. Results in characteristics polynomial of the form $-\lambda^{3}+a_{1} \lambda^{2}+a_{2} \lambda^{1}+a_{3}=0$ where $a_{1}, a_{2}, a_{3}$, depend the various combinations of the model parameter values. In this case, the three eigenvalues could all be negative, positive, zero, or any combinations of the three alternatives. Thus, the endemic equilibrium could be stable, unstable, or saddle depending on the values of the various model population parameters combined at a given time.

\section{RESULTS AND DISCUSSION}

The system of equation (2.1) was solved numerically using maple mathematical package. The hypothetical value for each of the model population parameters is as given in the table that follows:

Table 2: Hypothetic values of each model parameters used for the numerical solution.

\begin{tabular}{|c|c|}
\hline Parameter & Values \\
\hline N & 100 \\
\hline$?$ & 0.9 \\
\hline f & 0.50 \\
\hline
\end{tabular}

\begin{tabular}{|c|c|}
\hline $\mathrm{T}_{\mathrm{L}}$ & 0.8 \\
\hline $\mathrm{T}_{\mathrm{A}}$ & 0.74 \\
\hline $\mathrm{a}$ & 0.41 \\
\hline $\mathrm{d}$ & $1 /(365 \times 70)$ \\
\hline $\mathrm{p}$ & 0.00196 \\
\hline $\mathrm{e}$ & 0.0735 \\
\hline$\beta_{\mathrm{A}}$ & 0.01 \\
\hline
\end{tabular}

The results from our numerical solutions are displayed in the graphs that follow:

Graph of model Population againat the

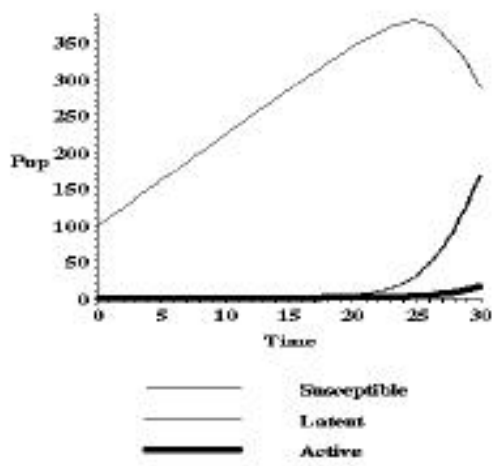

Fig. $1: \mathrm{S}(0)=99, \mathrm{I}_{\mathrm{L}}(0)=1, \mathrm{I}_{\mathrm{A}}(0)$.

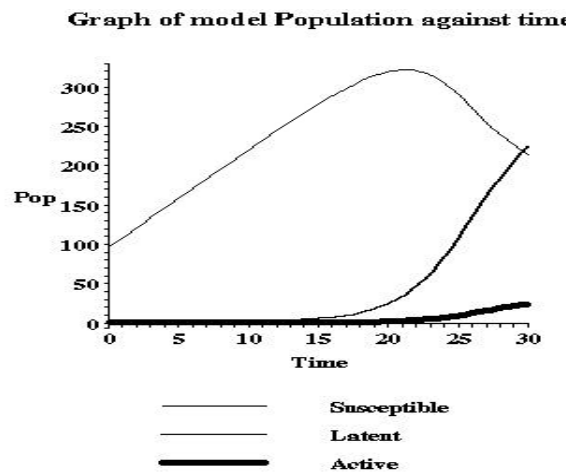

Fig. 2: $\mathrm{S}(0)=99, \mathrm{I}_{\mathrm{L}}(0)=0, \mathrm{I}_{\mathrm{A}}(0)=1$, Graph of model Population against time

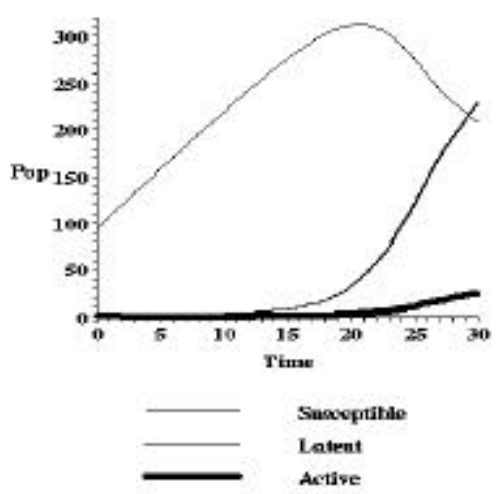

Fig.3: $\mathrm{S}(0)=96, \mathrm{I}_{\mathrm{L}}(0)=3, \mathrm{I}_{\mathrm{A}}(0)=1$. 
In Fig. 1-3 above, we solved the model equations and simulated for diffe rent initial conditions. The trivial case is when the initial condition were zeros for the latently and actively TB infected population, and for this we expect a steady rise in the susceptible with occasional drops while the infected (both latent and active) population remained at zero level. However, we did not bother to show the graph of this since it is obvious.

In Fig. 1, the TB spread starts with an latently infected patients, it takes sometime before the hitherto uninfected people in the environment gets infected, while in Fig. 2 the TB spread starts from an actively infected TB patients, the disease spread is very rapid leading to more latently infected people and less actively infected people. However, in Fig. 3 we considered a situation where the spread starts from both actively and latently infected TB patients, the results here shows no significant difference to the case when it starts from an actively infected patient except that the disease spread is slower in the latter.

In addition, we simulate our model equations for varying TB incidence transmission rates and below are the resulting graphs:

Graph of model Papulation againat time

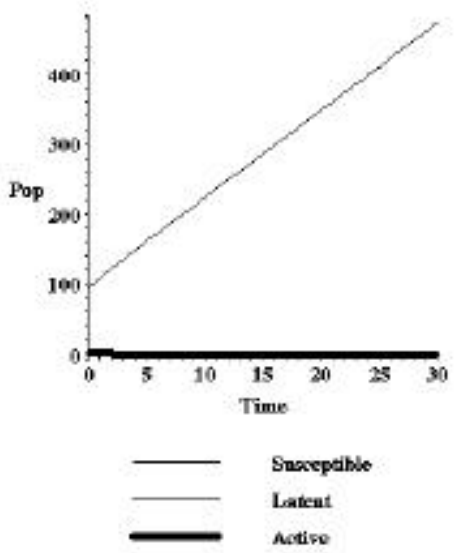

Fig. 4: Graphs of result for $\mathrm{a}=0.001$ Graph of model Population againat time

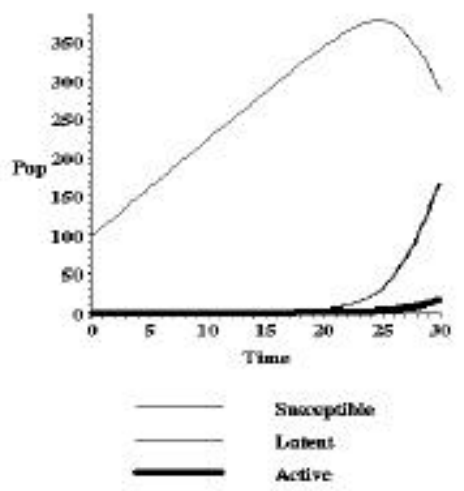

Fig. 5: Graphs of results for $a=0.041$
Graph of model Population sgainst time

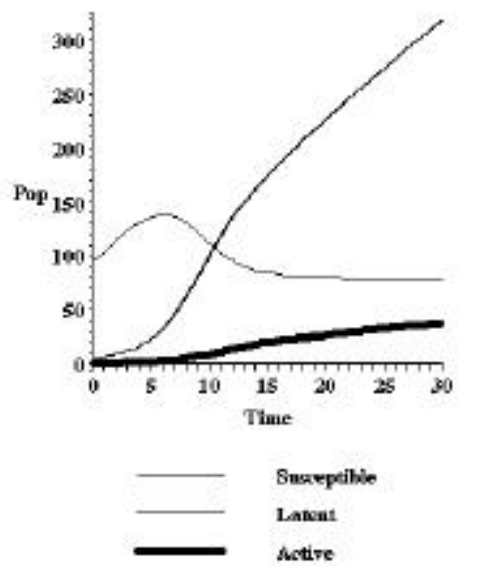

Fig. 6: Graphs of results for $a=0.1$

As can be seen from above graphs in Fig. s 4 to 6 , the disease spreads more rapidly as the disease incidence transmission rate increases. Precisely, when the disease incidence transmission rate (a) is 0.001 , meaning that one in every one thousand people that are exposed to the disease gets infected, the population of actively and latently infected remained close to zero all through. Moreover, when the disease incidence transmission rate (a) is 0.041 , meaning that about four in every one hundred people that are exposed to the disease gets infected, the population of latently and actively infected people starts to increase departing from zero with the former increasing more rapidly. Nevertheless, for a situation with disease incidence transmission rate (a) equals 0.1 , meaning that in every ten people that are exposed to the disease one gets infected, the disease spread more rapidly to the extent that within a short time, the number of latently infected people out-numbers the susceptible while the number of actively infected people approaches half the number of the susceptible. The inference that could be drawn from the above is that the spread of the disease can be drastically reduced if we can put in place measures and controls that will reduce the disease incidence transmission rate. Thus, any measures that will reduce either the respiratory contact with infection per susceptible or the probability that the contact leads to infection will help check the spread of the disease. Hence, this also confirms the findings of Gammaitoni and $\mathrm{Nucci}^{[3]}$ which asserts that administrative control measures are the most effective since they are capable of reducing the disease incidence transmission rate to as close as possible to zero. 


\section{CONCLUSIONS}

\section{REFERENCES}

In this research work, we considered the dynamics of the Tuberculosis disease population using the SIRS model. The resulting model equations were solved numerically and results were presented graphically based on our simulations. Our results show that the population dynamics depends more on the number of actively infected people in the population at the initial time and also on the disease incidence transmission rate at a given time. Most importantly, we also showed that the disease-free equilibrium is stable while the endemic equilibrium may or may not be stable depending on the various values of the model parameters.
1. Castro, K.G., 1998. Global Tuberculosis Challenges. Emerging Infectious Disease, 4(3).

2. Dye C, et al, 2000. Evaluating the impact of tuberculosis control number of deaths prevented by short-course chemotherapy in China. International Journal of Epidemiology, 29:558-564.

3. Gammaitoni L. and Nucci M.C., 1997. Using a mathematical model to evaluate the efficacy of TB control measures. Emerging Infectious Diseases, 3(3).

4. Murray C.J. and Salomon J.A., 1998. Using Mathematical models to evaluate global tuberculosis control strategies - A paper presented at the Centre for Population and Development studies, Harvard University, USA. (Unpublished )

5. World health Organization, 2007. WHO: Tuberculosis fact sheet, No 104. 